\title{
The utility of cardiopulmonary exercise testing in evaluating the efficacy of treatment in COPD
}

\author{
Sophie Lynne Corriveau \\ Natya Raghavan \\ 1 Department of Medicine, McMaster University, \\ Montreal, Canada
}

Address for correspondence:

Sophie Lynne Corriveau

Department of Medicine

McMaster University, Montreal, Canada

E-mail: sophie.corriveau@medportal.ca

\section{Summary}

Exercise tolerance is one of the major complaints from patients with chronic obstructive pulmonary disease (COPD) and has significant impact on perceived quality of life. Although the etiology of dyspnea in COPD is multifactorial, dynamic hyperinflation during exercise is a major contributor. Although $\mathrm{FEV}_{1}$ has served as an important diagnostic tool, it does not correlate well with patient's response or exercise performance. The 6 minute walk test is also problematic and has limited use in assessing patient's response to treatment since the test does not involve a fixed exercise stimulus. Inspired capacity, as a marker of dynamic hyperinflation, measured at various time points during constant load exercise testing does seem to correlate well with exercise endurance time as well as the patient's level of dyspnea making it a valuable tool to assess the response of various therapeutic modalities used in COPD. In this review we look at the impact of a broad range of therapies used in COPD and their effects on exercise. Bronchodilators, inhaled corticosteroids, oxygen, pulmonary rehabilitation and lung volume reduction surgery all reduce dynamic hyperinflation and improve important patient outcomes.

KEY WORDS: COPD, cardiopulmonary exercise testing, dynamic hyperinflation.

\section{Introduction}

Chronic obstructive pulmonary disease (COPD) is a common respiratory disorder which is characterized by airflow limitation and dysfunction of both large and small airways. It is associated with destruction of the lung parenchyma and its vasculature in variable combinations.

Exercise limitation represents an important feature of
Alleviation of symptoms of dyspnea and leg discomfort and improvement in activity level are important therapeutic goals. COPD which negatively impacts patients quality of life. Alleviation of symptoms of dyspnea and leg discomfort and improvement in activity level are important therapeutic goals. Although historically, the forced expiratory volume in 1 second $\left(F E V_{1}\right)$ has been used as a marker of disease severity, it correlates poorly with symptoms and function and does

not necessarily change with treatment (1). The aim of this paper is first to review the importance of assessing exercise capacity in COPD and the physiological impact of COPD on exertion as well as the variety of exercise tests available to clinicians at this time. Finally, we will review the literature on the impact of a broad range of therapies commonly used in the treatment of COPD on exercise parameters.

\section{Exercise limitation in COPD}

Exercise limitation occurs insidiously in patients with COPD. Detecting dyspnea is difficult because patients tend to modify their activities to avoid dyspnea (2). Furthermore, patients with COPD typically present when there has already been a significant decline in $\mathrm{FEV}_{1}$ accompanied by some degree of hyperinflation and exertional dyspnea. Even patients with mild COPD have been found to have profound physiological effects when the respiratory system is put under stress by exercise (3). Exercise intolerance in COPD is multifactorial and includes a reduced ventilatory capacity, increased work of breathing, skeletal muscle dysfunction due to deconditioning and possibly skeletal muscle apoptosis due to systemic inflammation, and destruction of the pulmonary capillary bed by emphysema (2) (Figure 1). Intolerable dyspnea and leg fatigue are typically the most common exercise limiting symptoms (4).
Abnormal dynamic ventilatory mechanics and excessive chemostimulation during exercise results in a widening disparity between escalating central neural drive and restricted thoracic volume displacement. 


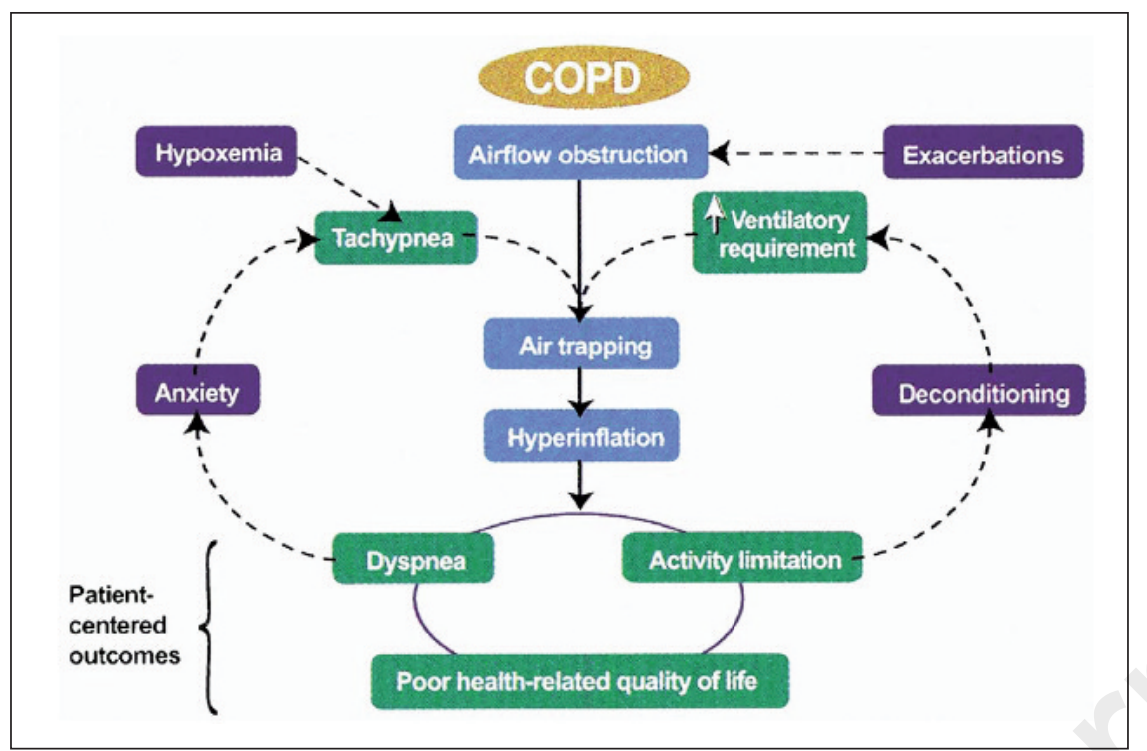

Figure 1 - Central role of air trapping and hyperinflation in the pathophysiology of chronic obstructive pulmonary disease (COPD). Although related to increased airways resistance, hyperinflation correlates more directly with patient-centered outcomes. Activity limitation leads to deconditioning, which, in turn, increases ventilator requirements, establishing a cycle of decline leading to worsening hyperinflation. Anxiety and hypoxemia cause tachypnea, which worsens hyperinflation by allowing less time for exhalation [Reprinted with permission from Am J Med (2)].

During exercise in normal subjects, end expiratory lung volume (EELV) remains stable or decreases as exercise increases. The end inspiratory volume (EILV) increases and tidal volume increases substantially. This is in contrast to patients with COPD where, as ventilatory demands increase in the setting of expiratory flow limitation, there is progressive air trapping and further dynamic hyperinflation (DH) above already increased resting values as depicted in Figure 2. EELV increases and as exercise continues there is progressively less inspiratory capacity (IC) available as EELV increases toward total lung capacity (TLC). The increase in EELV places the respiratory muscles at a mechanical disadvantage since the elastic work of breathing is increased owing to higher lung volumes and the need to counteract the greater inward recoil of the chest wall leading to the uncomfortable sensation of dyspnea (2). Abnormal dynamic ventilatory mechanics and excessive chemostimulation during exercise results in a widening disparity between escalating central neural drive and restricted thoracic volume displacement (5). This has been termed neuromechanical uncoupling which is thought to form the basis for the distressing sensation of an unsatisfied inspiration as often occurs in COPD (5).

Measurement of symptoms, operational lung volumes, spirometry and endurance during exercise in patients with COPD

There is increasing evidence that the IC during exercise, and the rate of change in IC during exercise (dynamic hyperinflation) are strong predictors of exertion- al dyspnea intensity and exercise intolerance; both of which are important patient-centered outcomes (6). The extent of dynamic hyperinflation during exercise varies inversely with the level of resting hyperinflation. Also, for a given degree of airflow obstruction, patients with lower diffusion capacity appear to have faster rates of dynamic hyperinflation (7). Although the $\mathrm{FEV}_{1}$ is used in the diagnosis and physiological staging of COPD; it has limited application in assessing the response to bronchodilators or predicting patient-centered outcomes in terms of exercise ability or dyspnea (2). Data from the National Emphysema Treatment Trial (NETT) demonstrated that for any $\mathrm{FEV}_{1}$, there was a wide range of maximum exercise capacity observed illustrating the limited predictive value of $F E V_{1}$ for exercise capacity in individual patients (8).

In COPD, the best physiological correlate of peak oxygen uptake $\left(\mathrm{Vo}_{2}\right)$ is the peak tidal volume which in turn is determined primarily by the peak IC during exercise or the resting IC (7). Exertional dyspnea also correlates best with constraints on tidal volume. There is a clear statistical association between the level of resting and dynamic hyperinflation, the degree of tidal volume restriction during exercise, and peak exercise performance (7). When the tidal volume during exercise ap-
There is a clear statistical association between the level of resting and dynamic hyperinflation, the degree of the tidal volume restriction during exercise, and peak exercise performance. proximates the peak IC, further volume expansion is nearly impossible despite further increase in inspiratory muscle effort. In a mul- 


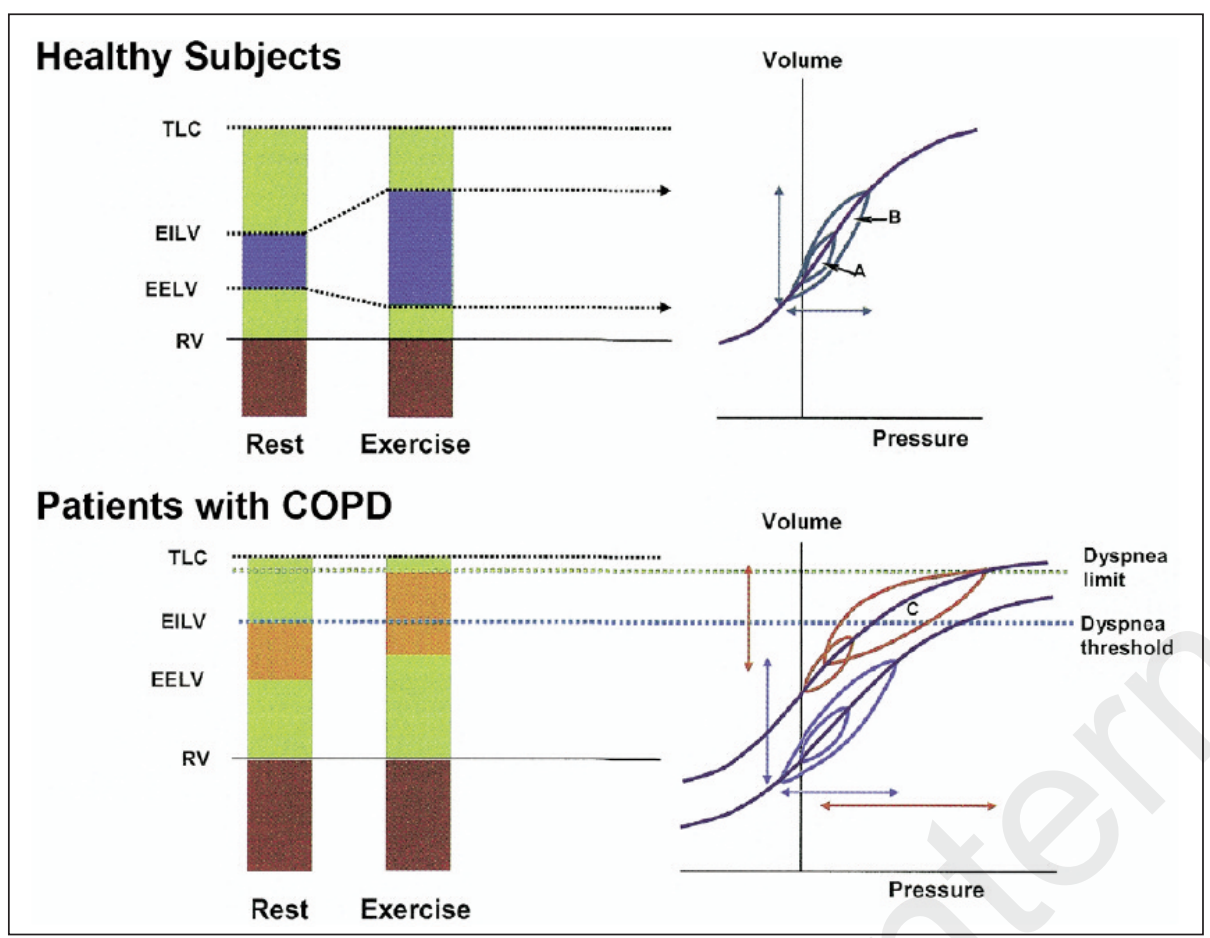

Figure 2 - Operational lung volumes at rest and with exercise in healthy subjects and in patients with chronic obstructive pulmonary disease (COPD). On the compliance curve of healthy subjects, the small ellipse (A) represents the volume at rest, and the large ellipse (B) represents the volume during exercise. Similarly, on the compliance curve of patients with COPD, the small ellipse on the upper curve represents the volume at rest, while the large ellipse $(C)$ on the upper curve represents the volume during exercise. The 2 ellipses on the lower curve represent the volume at rest and during exercise in healthy subjects for comparison. For the patients with COPD, the increase in end-expiratory lung volume (EELV) during exercise represents dynamic hyperinflation. The hypothetical "dyspnea threshold" and "dyspnea limit" are also shown. EILV=end-inspiratory lung volume; $\mathrm{RV}=$ residual volume; $\mathrm{TLC}=$ total lung capacity [Reprinted with permission from Am $\mathrm{J} \mathrm{Med} \mathrm{(2)].}$

tiple regression analysis with symptom limited peak $\mathrm{Vo}_{2}$ as the dependent variable and several relevant physiological measurements as independent variables, including $\mathrm{FEV}_{1}$ and $F E V_{1} / F V C$ ratio, peak tidal volume $\left(\mathrm{V}_{\mathrm{T}}\right)$ emerged as the strongest contributory variable explaining $47 \%$ of the variance in exercise limitation which in turn correlated strongly with resting and peak dynamic IC. Volume constraints contributed disproportionately to both exercise intolerance and dyspnea in patients with COPD (7).

Using a randomized, double blind, two-period crossover design allowing patients to serve as their own control, O'Donnell et al. examined the reliability and reproducibility of a variety of cardiopulmonary responses during exercise testing to determine whether they can accurately predict symptomatic response (6). Reliability (or reproducibility) was established by analyzing baseline measurements taken at repeated visits in 29 patients with moderate to severe COPD. Responsiveness to treatment was assessed by comparing the acute effects of two treatments (single dose nebulized ipratropium bromide versus placebo over a three week period followed by crossing over to the alternate treatment for an additional 3 week period after a 2 to 7 day washout period). Exercise performance during constant load testing was assessed by looking at

symptom-limited peak endurance time, peak oxygen uptake $\left(\mathrm{Vo}_{2}\right)$ and peak minute ventilation (VE). Although each measurement showed excellent reproducibility; only exercise endurance time was both reliable and responsive to change. Serial IC measurements during exercise were reproducible and responsive, and changes in IC after bronchodilator therapy correlated best with changes in exertional dyspnea compared to any other resting or exercise parameter including $\mathrm{FEV}_{1}$ and FVC. An increase in IC (or reduction in dynamic hyperinflation) of about 0.3-0.4L during exercise translated into a clinically significant improvement in exercise en-

IC during exercise, and the rate of change in IC during exercise (dynamic hyperinflation) are strong predictors of exertional dyspnea intensity and exercise intolerance; both of which are important patientcentered outcomes. durance and reduction in exertional dyspnea (6).

\section{Exercise testing in COPD}

The six minute walk test (6MWT) is frequently used to assess the functional status of patients with COPD. 


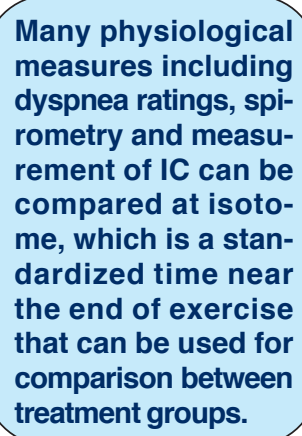
ference in perceived walking ability for patients with COPD is $54 \mathrm{~m}$ ( $\mathrm{Cl} 37$ to $71 \mathrm{~m})$ (9). As occurs during cardiopulmonary exercise testing (CPET), dynamic hyperinflation determined by measuring IC occurs in $6 \mathrm{MWT}(10)$. The 6MWT is problematic to assess treatment outcomes because the test does not involve a fixed exercise stimulus even when repeated in the same patient (2). It also does not allow the routine measurements of more detailed ventilator responses such as $\mathrm{IC}$, tidal volume and $\mathrm{VO}_{2}$ and $\mathrm{VCO}_{2}$ during exercise.

An endurance test performed at a submaximal constant work rate does offer the advantage of a fixed exercise stimulus (2). Both incremental testing and constant load endurance testing have been used to assess therapeutic responses in patients with COPD. Whereas incremental testing generally begins with 3 min of rest followed by 3 min of unloaded pedalling then an incremental phase of $5-25 \mathrm{~W} / \mathrm{min}$ until the patient reaches exhaustion; constant load endurance testing is similar but the work rate is adjusted to $75 \%$ of Wmax (highest work rate subject could maintain for $\geq 30$ s) (11). Increasingly, investigators are using constant load endurance testing at a standardized submaximal work load to evaluate the impact of interventions because it is highly sensitive in measuring acute changes in exercise endurance following various therapies and is more sensitive than other exercise modalities including 6MWT (12). The downside is that you require an initial incremental test to establish the Wmax, however subsequently the endurance test is more responsive to change.

The exercise endurance time is a sensitive measure and is used as an outcome measure when assessing various treatment modalities. It refers to the duration of loaded pedaling from the commencement of pedaling to the symptom limited endpoint where the patient indicates they need to stop or when they can no longer maintain the required pedaling rate (13). The minimum clinically relevant improvement (MCID) in endurance time was previously reported by PuenteMaestu et al. to be 101 seconds (14). Improved exercise performance should be reflected in a prolonged endurance time for exactly the same stimulus after intervention. Many physiological measures including dyspnea ratings, spirometry and measurement of IC can be compared at isotome, which is a standardized time near the end of exercise that can be used for comparison between treatment groups.

\section{Therapies used in COPD and their effect on exer- cise}

\section{Bronchodilators}

In clinical practice, bronchodilators are recommended for symptomatic relief in COPD patients with all stages of severity. Various Authors have examined the relationships between lung hyperinflation, respiratory discomfort and exercise intolerance in patients with COPD following treatment with bronchodilators which include both beta agonist and anticholinergic medications.

Belman et al. assessed the effects of bronchodilator induced changes on dynamic lung volumes in 13 patients with moderate to severe COPD in a blinded, randomized, cross-over study. Using incremental exercise testing, patients received 3 puffs of placebo or 3 puffs of albuterol, a short acting beta agonist, via MDI and then on subsequent visits received the alternate therapy. Following treatment with albuterol, Borg dyspnea rating scale improved by 1.4 units, which exceeds the MCID of 1 unit. Although $\mathrm{FEV}_{1}$ and FVC tended to improve following bronchodilatation; neither was associated with improvement in breathless-
Bronchodilators may and during exercise likely as a result of increased tidal expiratory flow rates and lung emptying due to enhanced airway patency following bronchodilator therapy. increase IC at rest

ness. Instead, the best predictor of breathlessness was a reduction in dynamic hyperinflation (1). These findings were similar to another study examining the effects of salmeterol, a long acting beta agonist, versus placebo in 23 patients with moderate to severe COPD using constant load exercise testing (15). In this study, salmeterol resulted in improved lung function and increased expiratory flow rates. EELV reduced by about $0.32 \mathrm{~L}$ with an associated increase in IC by $0.33 \mathrm{~L}$. The greater IC led to improved tidal volume in the salmeterol group. Symptom limited exercise endurance time improved by $1.6 \mathrm{~min}$ following treatment with salmeterol. Exertional dyspnea also tended to improve in the salmeterol group and correlated best with increased tidal volume, dynamic IC as well and resting IC (15).

Tiotropium is a long acting anticholinergic which has been shown to achieve sustained bronchodilatation and to improve quality of life in patients with COPD. It was studied by O'Donnell et al. in a multicentre double blind placebo controlled trial to evaluate its effects on exercise endurance (12). 187 patients with stable moderate-severe COPD were randomized to receive tiotropium $18 \mathrm{mcg}$ daily versus placebo. Exercise endurance time increased significantly from baseline in the treatment group after the first dose with further increases after 21 days and 42 days of treatment. There was a mean difference in endurance time of $67 \mathrm{~s}$ between tiotropium and placebo after 21 days of treatment and 105 s after 42 days of treatment. Those in the tiotropium group were also able to exercise longer 
before reaching the same degree of dyspnea. These findings were sustained over a 6 week period. Operating lung volumes at rest and throughout exercise were decreased. A correlation analysis showed close interrelationships between improved IC, reduced exertional dyspnea and improved endurance time during treatment with tiotropium. Although, tiotropium also resulted in consistent improvements in $\mathrm{FEV}_{1}$ compared to placebo, as in previous studies, this did not correlate with dyspnea ratings or exercise endurance time (12). Indacaterol, a novel ultra-long acting B2 agonist providing $24 \mathrm{hr}$ bronchodilatation was compared to placebo in patients with moderate-severe COPD. After 3 weeks of treatment, patients in the treatment group had an increased exercise endurance time of $111 \mathrm{~s}$ (16). These findings were particularly apparent in patients with more severe COPD (FEV $1<50 \%$ predicted) where the exercise time was 229s higher in the treatment group. The end exercise IC was significantly higher with indacaterol than with placebo both on day 1 and after 3 weeks of therapy with differences of 190 $\mathrm{mL}$ and $280 \mathrm{~mL}$ respectively. Although there was no significant difference between treatment groups in Borg dyspnea scores at the end of exercise, patients were able to endure constant work rate for longer before experiencing equivalent levels of dyspnea.

These are but a few studies which provide further support that exercise performance in COPD patients with and without bronchodilator therapy is determined in part by the degree of lung hyperinflation and the associated mechanical restriction at rest. The increased IC at rest and during exercise likely occurs as a result of increased tidal expiratory flow rates and lung emptying due to enhanced airway patency following bronchodilator therapy (12).

\section{Inhaled corticosteroids}

It is recommended that patients with moderate-severe COPD who are prone to exacerbations be treated with an inhaled corticosteroid (ICS) in combination with a long acting bronchodilator (17). ICS monotherapy is typically avoided given its association with pneumonia (18). Guenette et al. assessed whether the addition of fluticasone propionate 500ug twice daily in patients with moderate to severe COPD led to an improvement in dyspnea and exercise endurance time using a constant load cycle protocol. Seventeen subjects were enrolled in the study; all of whom were on baseline inhaled long-acting bronchodilators. Following 2 weeks of treatment with fluticasone propionate, exercise endurance time improved by an average of $188 \mathrm{~s}$ when compared to placebo exceeding the current MCID of 100 s. This however was associated with an improvement in standardized dyspnea ratings in only 10 of the 17 patients under study. There was variability among subjects in their response to treatment. Specifically, 2 subjects had unusual increases in endurance time whereas one had an unusual reduction in endurance time. Removal of these three outliers reduced the observed improvement in exercise endurance time to 152s however improved statistical significance. Operating lung volumes were consistently reduced at rest and throughout exercise in the fluticasone group. Although the residual volume (RV), total lung capacity (TLC) and functional residual capacity (FRC) were reduced by about $0.3 \mathrm{~L}$ there was no reciprocal increase in vital capacity (VC) and inspiratory capacity (IC). Instead there was a net reduction in TLC and an improvement in $\mathrm{FEV}_{1} / \mathrm{FVC}$ ratio. This is in contrast to results of previous studies looking at the effects of bronchodilators where the ratio is unchanged but the IC improves. Also in this study, $\mathrm{FEV}_{1}$ improved by $115 \mathrm{ml}$ following 2 weeks of fluticasone which is large and out of keeping with results from other similar studies. Nonetheless, spirometric improvements were not associated with increases in endurance time (16). Improvements in exercise endurance, although statistically significant were highly variable and the mechanism by which this occurs with inhaled corticosteroids is unclear.

\section{Phosphodiesterase inhibitors}

Roflumilast, a phosphodiesterase-4 inhibitor should be considered for patients with moderate to severe COPD with chronic bronchitis and a history of at least one exacerbation in the previous year to prevent acute exacerbations of COPD (17). Roflumilast seems to work as an anti-inflammatory agent and has no direct effects on airway smooth muscle (19). In a 12 week study conducted by O'Donnell et al. to assess the effects of roflumilast in patients with COPD and underlying lung hyperinflation; patients treated with roflumilast had no significant change in static lung hyperinflation, exercise endurance time or dyspnea ratings. Roflumilast however did seem to prevent worsening dynamic lung hyperinflation during exercise as occurred in those randomised to placebo. $\mathrm{FEV}_{1}$ did improve by an average of $84 \mathrm{~mL}$ in the roflumilast group however this did not translate into improved exercise tolerance or dyspnea rating. Given the lack of improvement in IC (or decrease in dynamic hyperinflation), it is not surprising that endurance time was not increased when compared to placebo (19).

\section{Oxygen}

O'Donnell et al. studied 11 clinically stable hypoxemic patients with advanced COPD comparing the acute effects of room air and $60 \%$ oxygen on various parameters during constant load exercise testing (10). Oxygen improved both dyspnea and exercise tolerance in these patients. Endurance time increased significantly by an average of 4.7 minutes. Borg-time ratings of both dyspnea and leg discomfort fell significantly in those receiving oxygen. There was a modest reduction in ventilation by about $3 \mathrm{~L} / \mathrm{min}$ achieved by a reduction in breathing frequency. Hyperoxia also resulted in a delay in dynamic hyperinflation during exercise and a reduction in operational lung volumes of about 
$0.3 \mathrm{~L}$ which resulted in improvement in important patient-centered outcomes (10).

A similar study was conducted by Somfay et al. however subjects consisted of ten clinically stable patients with COPD who were not hypoxemic (oxygen saturation $>92 \%$ at rest and $>88 \%$ during exercise) (20). During five subsequent constant work rate tests, subjects breathed compressed air, 30\%, 50\%, $75 \%$ or $100 \%$ oxygen in a random

Increasing $\mathrm{FiO}_{2}$ to 0.3 in hypoxemic COPD patients produced the most substantial changes in exercise tolerance, the sensation of dyspnea and the physiologic responses to exercise. Increasing further produced smaller changes, but there is the real concern that may lead to hypercapnia in patients with COPD. ratings and operational lung volumes accompanied this improvement. Increasing $\mathrm{FiO}_{2}$ to 0.3 produced the most substantial changes in exercise tolerance, the sensation of dyspnea and the physiologic responses to exercise whereas increasing further to an oxygen concentration of $50 \%$ produced an additional but smaller change. Increasing the concentration of oxygen above $50 \%$ had no further impact on exercise endurance or breathlessness (20). Although changes in operating lung volumes could account for a portion of variance in exercise tolerance or dyspnea; there are likely other contributing factors such as improved muscle function.

Although these and other similar studies provide evidence that supplemental oxygen compared to placebo increases exercise capacity in patients performing single-assessment exercise tests; there is little support for oxygen supplementation for exercise training in nonhypoxemic patients with COPD (21). There is also the real concern that uncontrolled oxygen may lead to hypercapnia in patients with COPD through worsening ventilation-perfusion mismatch, Haldane effect and hypoventilation (22). The first study demonstrated an increase in arterial $\mathrm{PCO}_{2}$ on $60 \%$ oxygen (53.3 vs $57.6 p<0.1$ ), which is especially significant given the short duration of treatment (22).

\section{Lung volume reduction surgery}

Several clinical trials have examined the benefits of lung-volume reduction surgery (LVRS) in selected patients with upper-lobe predominant emphysema and hyperinflation. Overall, LVRS results in improved static pulmonary function, improved functional status and decreased breathlessness and reduced dynamic hy- perinflation $(23,24)$. Exercise testing protocols did differ between studies and did not involve the more commonly used constant load exercise test but rather incremental exercise test or 6MWT to assess exercise endurance. Although one trial was identified that used CWR exercise testing, dynamic hyperinflation as expressed by IC was not measured until partway through the trial preventing sufficient power to reach statistical significance (24).

\section{Pulmonary rehabilitation}

Pulmonary rehabilitation has also been shown to improve exercise tolerance in patients with COPD by reducing the degree of hyperinflation at a given level of exercise. During CWR exercise, ventilation and breathing rate were lower after training. There was also a reduction in hyperinflation as reflected by a higher IC. The improvement in exercise tolerance correlated with the change in isotime IC suggesting that exercise training decreases ventilatory requirements and dynamic hyperinflation (25).

\section{Conclusion}

Exercise limitation is common in patients with COPD and is associated with a decreased quality of life. Among the multitude of symptoms faced by those with this disease, dyspnea is one of the most distressing with a great impact on quality of life and manifests as exercise intolerance. Although exercise intolerance is multifactorial, it appears to be tightly linked to volume constraints and the extent of dynamic hyperinflation that typically occurs in patients with COPD during exercise. Inspiratory capacity measured at rest, at a standardized level of submaximal exercise, and at peak exercise is highly reproducible and responsive to change (6). When compared to other physiological parameters measured during exercise, an increase in IC (or reduction in dynamic hyperinflation) of approximately 0.3 to $0.4 \mathrm{~L}$ during exercise translates into a clinically significant improvement in exercise endurance and reduction in exertional dyspnea. Given its reliability and responsiveness to therapy it is used to assess the effects of novel therapies in COPD. It also gives a more comprehensive physiological assessment of the patient than other tests such as spirometry or the 6MWT. We have reviewed here a number of the commonly used treatments in COPD and their physiological effects as evaluated by cardiopulmonary exercise testing. These studies help to illustrate the pathophysiology of dyspnea in COPD and the mechanisms of dyspnea relief in those treatments with proven benefit to exercise capacity. 


\section{References}

1. Belman MJ, Botnick WC, Shin JW. Inhaled bronchodilators reduce dynamic hyperinflation during exercise in patients with chronic obstructive pulmonary disease. Am J Respir Crit Care Med. 1996;153:967975.

2. Cooper CB. The connection between chronic obstructive pulmonary disease symptoms and hyperinflation and its impact on exercise and function. Am J Med. 2006;119:S21-S31.

3. Ofir D, Laveneziana P, Webb, KA, Lam YM, O'Donnell DE. Mechanisms of dyspnea during cycle exercise in symptomatic patients with GOLD stage I chronic obstructive pulmonary disease. Am J Respir Crit Care Med. 2008;177:622-9.

4. Laveneziana P, Webb KA, Ora J, Wadell K, O'Donnell $E$. Evolution of dyspnea during exercise in chronic obstructive pulmonary disease. Am J Respir Crit Care Med. 2011;184:1367-1373.

5. O'Donnell DE, et al. Pathophysiology of dyspnea in chronic obstructive pulmonary disease: a roundtable. Proc Am Thorac Soc. 2007;4:145-168.

6. O'Donnell DE, Lam M, Webb K. Measurement of symptoms, lung hyperinflation, and chronic endurance during exercise in chronic obstructive pulmonary disease. Am J Respir Crit Care Med. 1998;158:1557-1565.

7. O'Donnell DE, Revill SM, Webb KA. Dynamic hyperinflation and exercise intolerance in chronic obstructive pulmonary disease. Am J Respir Crit Care Med. 2001;164:770-777.

8. National Emphysema Treatment Trial Research Group. Patients at high risk of death after lung-volume-reduction surgery. N Engl J Med. 2001;345: 1075-1083.

9. Redelmeier DA, Bayoumi AM, Goldstein RS, Guyatt $\mathrm{GH}$. Interpreting small differences in functional status: the six minute walk test in chronic lung disease patients. Am J Respir Crit Care Med. 1997;155:12781282.

10. O'Donnell DE, D'Arsigny C, Webb KA. Effects of hyperoxia on ventilatory limitation during exercise in advanced chronic obstructive pulmonary disease. Am J Respir Crit Care Med. 2001; 163:892-898.

11. ATS/ACCP statement on cardiopulmonary exercise testing. Am J Respir Crit Care Med. 2003;167:211 277.

12. O'Donnell DE, Flüge T, Gerken F, Hamilton A, Webb $\mathrm{K}$, Aguilaniu $\mathrm{B}$, et al. Effects of tiotropium on lung hyperinflation, dyspnea and exercise tolerance in COPD. Eur Respir J. 2004;23:832-840.

13. Guenette JA, Raghavan N, Harris-McAllister V, Preston ME, Webb KA, O'Donnell DE. Effect of adjunct fluticasone propionate on airway physiology during rest and exercise in COPD. Resp Med. 2011;105: 1836-1845.

14. Puente-Maestu L, Villar F, de Miguel J, Stringer WW, Sanz P, Sanz ML, et al. Clinical relevance of constant power exercise duration changes in COPD. Eur Respir J. 2009;34:340-345.

15. O'Donnell DE, Voduc N, Fitzpatrick M, Webb KA. Effect of salmeterol on the ventilatory response to exercise in chronic obstructive pulmonary disease. Eur Respir J. 2004;24:86-94.

16. O’Donnell DE, Casaburi R, Vincken W, PuenteMaestu L, Swales J, Lawrence D, et al. Effect of indacaterol on exercise endurance and lung hyperinflation in COPD. Resp Med. 2011;105:1030-1036.

17. Criner GJ, Bourbeau J, Diekemper RL, Ouellette DR, Goodridge D, Hernandez P, et al. Executive summary: prevention of acute exacerbations of COPD: American College of Chest Physicians and Canadian Thoracic Society Guideline. Chest. 2015; 147:883-893.

18. Calverley PM, Anderson JA, Celli B, Ferguson GT, Jenkins $C$, Jones PW, et al. Salmeterol and fluticasone propionate and survival in chronic obstructive pulmonary disease. New Engl J Med. 2007;356:775789.

19. O’Donnell DE, Bredenbroker D, Brose M, Webb KA. Physiological effects of roflumilast at rest and during exercise in COPD. Eur Respir J. 2012;39:1104-1112.

20. Somfay A, Porszasz J, Lee SM, Casaburi R. Doseresponse effect of oxygen on hyperinflation and exercise endurance in nonhypoxaemic COPD patients. Eur Respir J. 2001;18:77-84.

21. Nonoyama M, Brooks D, Lacasse Y, Guyatt GH, Goldstein R. Oxygen therapy during exercise training in chronic obstructive pulmonary disease (review). The Cochrane Collaboration. 2007, Issue 2.

22. Abdo WF \& Heunks LMA. Oxygen induced hypercapnia: myths and facts. Critical care. 2012;16:323326.

23. Martinez FJ, Montes de Oca M, Whyte RI, Stetz J, Gay SE, Celli BR. Lung volume reduction improves dyspnea, dynamic hyperinflation, and respiratory muscle function. Am J Respir Crit Care Med. 1997;155:1984-1990.

24. Dolmage TE, Waddell TK, Maltais F, Guyatt GH, Todd TRJ, Keshavjee S, et al. The influence of lung volume reduction surgery on exercise in patients with COPD. Eur Respir J. 2004;23:269-274.

25. Porszasz J, Emtner M, Goto S, Somfay A, Whipp BJ, Casaburi R. Exercise training decreases ventilatory requirements and exercise-induced hyperinflation at submaximal intensities in patients with COPD. Chest. 2005;128:2025-2034. 